\title{
Some features of metal cerium asymptotical behavior
}

\author{
E.D.Soldatova, T.E.Galachenko \\ Dnipropetrovs'k National University
}

Received July 18, 2005, in final form December 19, 2005

\begin{abstract}
The relations between the critical exponents of basic stability characteristics are obtained based on the thermodynamic stability requirements for the generalized one-component system without engaging any hypotheses. The conditions are obtained, where these relations are fulfilled as equalities. The outcomes are applied to the consideration of critical properties of the isomorphic $\boldsymbol{\gamma}-\boldsymbol{\alpha}$ transition in the metal cerium. It is shown, that for cerium the corresponding relations are fulfilled as equalities. It is also shown, that the equilibrium curve in the $P-T$ phase diagram of cerium continuously passes into the lowered stability curve in the supercritical region, that provides symmetry between its critical exponents. The obtained results are in accord with the scaling law hypothesis without any assumptions about homogeneous functions for the potentials.
\end{abstract}

Key words: stability coefficients, critical exponents, statical scaling law hypothesis, phase equilibrium curve, quasispinodal

PACS: $64.60 . F r$

\section{Introduction}

One of the problems of the modern phenomenological theory of a critical state is the establishment of universal relations between critical exponents in the vicinity of the critical point. These relations are obtained by various methods, often by using various hypotheses. Naturally the following question arises: how can these relations be obtained based on the general thermodynamic method? In [1] it was shown, that the basic relations between the critical exponents (for example, the relations by Griffiths, Rushbrooke, Coopersmith etc.) follow from the first principles, from general aspects of the thermodynamic stability theory without engaging any hypotheses.

The new results are obtained in the suggested consideration by means of the thermodynamic method of stability [1] for the generalized homogeneous system. They were used for the investigation of the asymptotical behavior of metal cerium. Therefore we shall focus on some general aspects of the thermodynamic stability theory [2,3], which are indispensable for further consideration, as well as perform a few mathematical transformations.

\section{Elements of the stability theory. Some relations}

Let us consider the generalized one-component homogeneous system in the state determined by the internal energy $U=U(S, x)$, where $S$ is the entropy, $x$ is a generalized thermodynamic variable, and $T$ and $X$ are the corresponding thermodynamic forces.

The stability of such a system is determined by the well-known requirements [3]:

$$
\begin{aligned}
D= & \frac{\partial(T, X)}{\partial(S, x)}=\left|\begin{array}{ll}
\left(\frac{\partial T}{\partial S}\right)_{x} & \left(\frac{\partial T}{\partial x}\right)_{S} \\
\left(\frac{\partial T}{\partial x}\right)_{S} & \left(\frac{\partial X}{\partial x}\right)_{S}
\end{array}\right|=\left(\frac{\partial T}{\partial S}\right)_{x}\left(\frac{\partial X}{\partial x}\right)_{S}-\left(\frac{\partial T}{\partial x}\right)_{S}^{2} \geqslant 0 \\
& \left(\frac{\partial T}{\partial S}\right)_{x} \geqslant 0,\left(\frac{\partial X}{\partial x}\right)_{S} \geqslant 0 .
\end{aligned}
$$


The value $D$, following Semenchenko V.K. [3], is called the determinant of stability, and the derivatives in it are the adiabatic parameters (the AP's); the parameters $\left(\frac{\partial T}{\partial S}\right)_{x},\left(\frac{\partial X}{\partial x}\right)_{S}$ are the adiabatic stability coefficients (the ASC's). The parameters $\left(\frac{\partial T}{\partial S}\right)_{X},\left(\frac{\partial X}{\partial x}\right)_{T},\left(\frac{\partial T}{\partial x}\right)_{X}$, measured for the constant thermodynamic forces, are the isodynamic parameters (the IP's).

The following relation between the AP's, the IP's and $D$ is obtained as the result of Jacobian properties:

$$
D=\left(\frac{\partial T}{\partial S}\right)_{x}\left(\frac{\partial X}{\partial x}\right)_{T}=\left(\frac{\partial T}{\partial S}\right)_{X}\left(\frac{\partial X}{\partial x}\right)_{S}=-\left(\frac{\partial T}{\partial x}\right)_{X}\left(\frac{\partial T}{\partial x}\right)_{S} .
$$

Using (1), we also obtain the following relations

$$
\begin{aligned}
& \left(\frac{\partial T}{\partial S}\right)_{x}\left(\frac{\partial S}{\partial T}\right)_{X}+\left(\frac{\partial T}{\partial x}\right)_{S}\left(\frac{\partial x}{\partial T}\right)_{X}=1 \\
& \left(\frac{\partial X}{\partial x}\right)_{S}\left(\frac{\partial x}{\partial X}\right)_{T}+\left(\frac{\partial T}{\partial x}\right)_{S}\left(\frac{\partial x}{\partial T}\right)_{X}=1 \\
& \left(\frac{\partial T}{\partial S}\right)_{x}\left(\frac{\partial x}{\partial T}\right)_{X}+\left(\frac{\partial T}{\partial x}\right)_{S}\left(\frac{\partial x}{\partial T}\right)_{X}=0 \\
& \left(\frac{\partial X}{\partial x}\right)_{S}\left(\frac{\partial x}{\partial T}\right)_{X}+\left(\frac{\partial T}{\partial x}\right)_{S}\left(\frac{\partial x}{\partial T}\right)_{X}=0 .
\end{aligned}
$$

We rewrite (3) using (2) in the form

$$
\begin{aligned}
& \left(\frac{\partial T}{\partial S}\right)_{x}\left(\frac{\partial S}{\partial T}\right)_{X}=\left(\frac{\partial X}{\partial x}\right)_{S}\left(\frac{\partial x}{\partial X}\right)_{T}=1-\left(\frac{\partial T}{\partial x}\right)_{S}\left(\frac{\partial x}{\partial T}\right)_{X}=1+\frac{\left(\frac{\partial T}{\partial x}\right)_{S}^{2}}{D} \\
& \left(\frac{\partial T}{\partial S}\right)_{x}\left(\frac{\partial x}{\partial T}\right)_{X}=-\left(\frac{\partial T}{\partial x}\right)_{S}\left(\frac{\partial x}{\partial X}\right)_{T}, \\
& \left(\frac{\partial X}{\partial x}\right)_{S}\left(\frac{\partial x}{\partial T}\right)_{X}=-\left(\frac{\partial T}{\partial x}\right)_{S}\left(\frac{\partial S}{\partial T}\right)_{X} .
\end{aligned}
$$

\section{Some relations between the critical exponents}

Let us consider the asymptotic behavior of the thermodynamic parameters upon approaching the critical point. We introduce the critical exponents of thermodynamic parameters, which determine the system stability considering the AP's and the IP's features as different.

$$
\begin{gathered}
\left(\frac{\partial T}{\partial S}\right)_{X} \sim \tau^{\alpha},\left(\frac{\partial X}{\partial x}\right)_{T} \sim \tau^{\gamma},\left(\frac{\partial T}{\partial x}\right)_{X} \sim \tau^{\mu} \\
\left(\frac{\partial T}{\partial S}\right)_{x} \sim \tau^{\alpha_{1}},\left(\frac{\partial X}{\partial x}\right)_{S} \sim \tau^{\gamma_{1}},\left(\frac{\partial T}{\partial x}\right)_{S} \sim \tau^{\mu_{1}} \\
\tau=\left|\frac{T-T_{C}}{T_{c}}\right| .
\end{gathered}
$$

Let us emphasize, that the introduced exponents $\mu$ and $\mu_{1}$ are more natural for thermodynamic stability consideration, than the order parameter exponent $\beta$. They enable us to consider the asymptotical behavior in the subcritical and supercritical regions. The relation between the AP's exponents can be derived by means of asymptotics (5) from the stability conditions (1):

$$
\alpha_{1}+\gamma_{1} \leqslant 2 \mu_{1}
$$


Using (2) and (5) we obtain the relation for the IP's critical exponents:

$$
\alpha+\gamma \geqslant 2 \mu
$$

Relations (6) and (7) are the most general form of relations and determine the correspondence between the AP's and the IP's critical exponents for the subcritical (the primed exponents below) and the supercritical vicinity of the critical point. These relations connect the basic thermodynamic parameters. For example, (6) connects the exponents for the isochoric heat capacity, the adiabatic compressibility and thermal broadening connect the exponents for the $P-V-T$ systems. Relation (7) binds the critical exponents for isobaric heat capacity, isothermal compressibility, and isobaric thermal broadening.

A significant task for the modern critical exponent phenomenological theory is to obtain equalities in the relations between the critical exponents. Thermodynamic hypothesis, which is based on the scaling law hypothesis (i.e., static scaling law hypothesis), leads to the equalities between the critical exponents [4]. This hypothesis is based on the assumption that the thermodynamic potentials are generalized homogeneous functions. This assumption results in the transformation of inequalities between critical exponents into equalities. Besides, the hypothesis leads to equalities between the critical exponents above the critical point and below it (symmetry between the critical exponents). A logical question arises regarding the condition, for which relations (6) and (7) are changed to equalities from the viewpoint of general requirements of the stability theory.

Let us approach the critical point, using asymptotics (5). Then from relations (4) it follows

$$
\alpha-\alpha_{1}=\gamma-\gamma_{1} \geqslant 0, \quad \alpha_{1}+\gamma=\mu+\mu_{1}, \quad \alpha+\gamma_{1}=\mu+\mu_{1} .
$$

These relations connect the AP's and the IP's exponents and they are correct independently of the product $\left(\frac{\partial T}{\partial x}\right)_{X}^{-1}\left(-\frac{\partial T}{\partial x}\right)_{S}$. Its value is essential for obtaining the following results.

The limit value of this product is

$$
C \equiv \lim _{D \rightarrow 0} \frac{-\left(\frac{\partial T}{\partial x}\right)_{S}}{\left(\frac{\partial T}{\partial x}\right)_{X}}=\lim _{D \rightarrow 0} \frac{\left(\frac{\partial T}{\partial x}\right)_{S}^{2}}{D} \geqslant 0 .
$$

For $C$ we have two possibilities:

1. If $C \neq \infty$, then $\mu<\mu_{1}$. According to (4), (5) we obtain

$$
\alpha=\alpha_{1}, \quad \gamma=\gamma_{1}, \quad \alpha+\gamma=\mu+\mu_{1} \geqslant 2 \mu .
$$

2. If $C=\infty$, then $\mu \geqslant \mu_{1}$ and according to (4), (5) we obtain

$$
\begin{aligned}
\frac{1}{2}(\gamma-\alpha) & =\frac{1}{2}\left(\gamma_{1}-\alpha_{1}\right)=\gamma-\mu=\gamma_{1}-\mu_{1}=\mu-\alpha=\mu_{1}-\alpha_{1} \\
\alpha-\alpha_{1} & =\gamma-\gamma_{1}=\mu-\mu_{1} \geqslant 0 \\
\alpha+\gamma & =2 \mu, \quad \alpha_{1}+\gamma_{1}=2 \mu_{1} .
\end{aligned}
$$

Within the framework of thermodynamics, it appears possible to describe two possibilities: the states with $\mu \geqslant \mu_{1}$, for which $\alpha+\gamma=2 \mu, \alpha_{1}+\gamma_{1}=2 \mu_{1}$ with fulfilling the relations (10), and the states with $\mu<\mu_{1}$, for which $\alpha+\gamma \geqslant 2 \mu, \alpha_{1}+\gamma_{1} \leqslant 2 \mu_{1}$ and relations (9) are true.

As it will be seen hereinafter, the concrete definition of practicability of these both cases is determined by the critical behavior type of the thermodynamic systems [5]. The existence of four alternative types of critical behavior has been established in paper [5] based on the critical state definition by means of a set of homogeneous linear equations and the analysis of the critical state stability conditions. The types of behavior are classified by the value of an equilibrium curve slope at the critical point $K_{\mathrm{c}}$ and by one of the ASC's - either $\left(\frac{\partial T}{\partial S}\right)_{x}$ or $\left(\frac{\partial X}{\partial x}\right)_{S}$. 


\section{The asymptotical behavior of metal cerium}

The outcomes can be applied to the vicinity of the isomorphic $\gamma-\alpha$ transition critical point in the metal cerium. It is necessary to consider the pressure $P$ as the generalized thermodynamic force $X$, and the volume $V$ as the coordinate $x$ for cerium under pressure. From the thermodynamic standpoint, cerium is a homogeneous one-component $P-V-T$ system.

The critical properties of cerium have been researched in detail in papers [6,7] during the investigation of perfected models $[9,10]$. It was shown, that the adiabatic stability coefficient $\left(\frac{\partial T}{\partial S}\right)_{V}=\frac{T}{C_{V}}$ is unequal to zero. Besides, the critical slope for cerium is finite $K_{\mathrm{c}} \neq\{0, \infty\}$ in the $P-T$ phase diagram $[8,9]$. Therefore, the critical properties of cerium relate to the first type of critical behavior according to thermodynamic classification [5]. In paper [5] it was shown, that for the first type of critical behavior all the AP's are finite and nonzero, and all the IP's tend to zero by the same law. In terms of (5) it means that $\alpha_{1}=\mu_{1}=\gamma_{1} ; \quad \alpha=\mu=\gamma$. The calculations, performed in [6], have shown, that $\alpha_{1}=\mu_{1}=\gamma_{1}=0$, and $\quad \alpha=\mu=\gamma=1$. I.e., for cerium in the vicinity of the critical point $\mu>\mu_{1}$ and this is the case when the relations (6) and (7) are fulfilled as equalities as well as the relations (10) are satisfied.

It is necessary to note that the above result is derived based on the stability conditions only, without engaging any hypotheses.

Let us consider the equalities fulfilling between the critical exponents for $T \rightarrow T_{\mathrm{c}}^{-}$and $T \rightarrow T_{\mathrm{c}}^{+}$, i.e. the symmetry conditions fulfilling between the critical exponents.

From the thermodynamic point of view this question is connected with the condition for transformation of the phase equilibrium curve in the subcritical region into the lowered stability curve in the supercritical region. Let us note that one of the basic ideas of the thermodynamic method of the critical state investigation [5] is the binding of subcritical and supercritical system properties at the critical point. According to [3] the supercritical phase properties also remain above the critical point. It leads to passing the stability characteristics through the finite dips (the lowered stability region). The minima positions of the stability characteristics are called the lowered stability curve (quasispinodal) [3]. Exactly such a behavior of stability characteristics for cerium is described in papers $[6,7]$ based on the computations for models $[9,10]$.

It is logical to suppose, that the symmetry between critical exponents will take place, if the phase equilibrium curve continuously passes into the lowered stability curve (quasispinodal).

Let us find the quasispinodal equation. Consider the ordinary one-component system under the pressure $P$. The stability determinant of such a system is

$$
D(S, V)=\frac{\partial(T,-P)}{\partial(S, V)}=\left(\frac{\partial T}{\partial S}\right)_{V}\left(-\frac{\partial P}{\partial V}\right)_{S}-\left(\frac{\partial T}{\partial V}\right)_{S}^{2} .
$$

For the quasispinodal $\mathrm{d} D=0$ and the following condition is satisfied

$$
\mathrm{d} D=\left(\frac{\partial D}{\partial S}\right)_{V} \mathrm{~d} S+\left(\frac{\partial D}{\partial V}\right)_{S} \mathrm{~d} V=0
$$

or

$$
\left(\frac{\partial D}{\partial S}\right)_{V}=0, \quad\left(\frac{\partial D}{\partial V}\right)_{S}=0
$$

The latter relations give

$$
\begin{gathered}
\left(\frac{\partial^{2} T}{\partial S^{2}}\right)_{V}\left(-\frac{\partial P}{\partial V}\right)_{S}-\left(\frac{\partial T}{\partial S}\right)_{V}\left(-\frac{\partial^{2} P}{\partial V \partial S}\right)-2\left(\frac{\partial T}{\partial V}\right)_{S} \frac{\partial^{2} T}{\partial V \partial S}=0 \\
\frac{\partial^{2} T}{\partial V \partial S}\left(-\frac{\partial P}{\partial V}\right)_{S}+\left(\frac{\partial T}{\partial S}\right)_{V}\left(-\frac{\partial^{2} P}{\partial V^{2}}\right)_{S}-2\left(\frac{\partial T}{\partial V}\right)_{S}\left(\frac{-\partial^{2} T}{\partial V^{2}}\right)_{S}=0
\end{gathered}
$$

This set of equations is satisfied, if

$$
\left(\frac{\partial^{2} T}{\partial S^{2}}\right)_{V}=\frac{\partial^{2} T}{\partial V \partial S}=-\frac{\partial^{2} P}{\partial V \partial S}=\left(-\frac{\partial^{2} P}{\partial V^{2}}\right)_{S}=\left(\frac{\partial^{2} T}{\partial V^{2}}\right)_{S}=0
$$


The projection of the quasispinodal on the $P-T$ plane, with the independent variables $S, V$ for $\mathrm{d} T / \mathrm{d} P$, has the following form:

$$
\begin{aligned}
\frac{\mathrm{d} T}{\mathrm{~d} P}= & \frac{\frac{\partial T}{\partial S} \mathrm{~d} S+\frac{\partial T}{\partial V} \mathrm{~d} V}{\frac{\partial P}{\partial S} \mathrm{~d} S+\frac{\partial P}{\partial V} \mathrm{~d} V}=f(S, V) \\
\mathrm{d} f= & \frac{\frac{\partial^{2} T}{\partial S^{2}} \mathrm{~d} S^{2}+2 \frac{\partial^{2} T}{\partial S \partial V} \mathrm{~d} S \mathrm{~d} V+\frac{\partial^{2} T}{\partial V^{2}} d V^{2}}{\frac{\partial P}{\partial S} \mathrm{~d} S+\frac{\partial P}{\partial V} \mathrm{~d} V}-\frac{\left(-\frac{\partial^{2} T}{\partial S \partial V}\right) \mathrm{d} S^{2}+2 \frac{\partial^{2} P}{\partial S \partial V} \mathrm{~d} S \mathrm{~d} V+\frac{\partial^{2} P}{\partial V^{2}} \mathrm{~d} V^{2}}{\left(\frac{\partial P}{\partial S} \mathrm{~d} S+\frac{\partial P}{\partial V} \mathrm{~d} V\right)^{2}} \\
& \times\left(\frac{\partial T}{\partial S} \mathrm{~d} S+\frac{\partial T}{\partial V} \mathrm{~d} V\right) .
\end{aligned}
$$

We used here the Maxwell's relation

$$
\left(\frac{\partial P}{\partial S}\right)_{V}=-\left(\frac{\partial T}{\partial V}\right)_{S}, \quad \frac{\partial^{2} P}{\partial S^{2}}=-\frac{\partial^{2} T}{\partial S \partial V}
$$

Substituting the condition (11) into (12), we have: $\mathrm{d} f=0$, i.e. for the $P-T$ plane the quasispinodal equation is

$$
\frac{\mathrm{d} T}{\mathrm{~d} P}=f(S, V)=\text { const. }
$$

Therefore, the quasispinodal in the $P-T$ plane is a straight line for any ordinary one-component $P-V-T$ system, including the supercritical region of cerium.

It is known [8-11], that the $P-T$ phase diagram of cerium has the feature that the phase equilibrium curve is a straight line. So, it is natural to expect a symmetry between the critical exponents, if in the $P-T$ phase diagram the phase equilibrium curve and quasispinodal continuously transforms one into another (without a break) at the critical point.

Indeed, according to the experiment [8] and to the models $[9,10]$, the equilibrium curve of $\gamma-$ and $\alpha$ - phases is a straight line with the slope $\mathrm{d} T / \mathrm{d} P=24.5 \mathrm{grad} / \mathrm{kbar}$ which is also kept at the critical point. It is evident from the model [9], that the quasispinodal is also a straight line with the slope $\mathrm{d} T / \mathrm{d} P=24.5 \mathrm{grad} / \mathrm{kbar}$.

Moreover, the critical exponents for the IP's and AP's have been found [6] for $T \rightarrow T_{\mathrm{c}}^{-}$, and $T \rightarrow T_{\mathrm{c}}^{+}$. It was shown, that $\alpha=\gamma=\mu=1\left(\right.$ for $\left.T \rightarrow T_{\mathrm{c}}^{+}\right) ; \alpha^{\prime}=\gamma^{\prime}=\mu^{\prime}=1\left(\right.$ for $\left.T \rightarrow T_{\mathrm{c}}^{-}\right)$; $\alpha_{1}=\gamma_{1}=\mu_{1}=0\left(\right.$ for $\left.T \rightarrow T_{\mathrm{c}}^{+}\right) ; \alpha_{1}^{\prime}=\gamma_{1}^{\prime}=\mu_{1}^{\prime}=0$ (for $T \rightarrow T_{\mathrm{c}}^{-}$). Thus $\alpha=\alpha^{\prime}, \alpha_{1}=\alpha_{1}^{\prime} ; \quad \gamma=$ $\gamma^{\prime}, \gamma_{1}=\gamma_{1}^{\prime} ; \mu=\mu^{\prime}, \mu_{1}=\mu_{1}^{\prime}$. This result enables us to conclude the existence of a symmetry between the corresponding exponents.

Hence, the available model and experimental data of the isomorphic $\gamma-\alpha$ transition in cerium are well agreed with the above-developed theoretical approach.

\section{Conclusions}

The obtained results can be reduced to the following

1. The relations between the critical exponents of isodynamic and adiabatic parameters have been obtained. They may be divided into two groups (9), (10) by the value of exponents $\mu$ and $\mu_{1}$. It is shown, that the strong equalities correspond to the case $\mu \geqslant \mu_{1}$, and the inequalities correspond to the case $\mu<\mu_{1}$.

2. Due to the realization of the first type of critical behavior by thermodynamic classification of critical states, the condition $\mu>\mu_{1}$ is satisfied and the relations (6), (7) are fulfilled as equalities for the metal cerium. 
3. It is shown, that the lowered stability curve in the $P-T$ phase diagram is a straight line.

4. In the metal cerium the phase equilibrium curve of the isomorphic $\gamma-\alpha$ transition passes continuously into the lowered stability curve (quasispinodal), which causes the symmetry between the critical exponents. This is confirmed by the experiment and models.

5. The obtained results correspond to the conclusions of the static scaling law hypothesis without engaging the hypothesis of homogeneous function for the potentials.

\title{
References
}

1. Soldatova E.D., Physical Chemistry Journal, 1984, 58, No. 12, 2913-2917 (in Russian).

2. Gibbs J., Thermodynamics. Statistical Mechanics. Moskow, Nauka, 1982 (in Russian).

3. Semenchenko V.K., Crystallography, 1964, 9, No. 5, 611-621 (in Russian).

4. Stanley H.E. The Phase Transitions and the Critical Phenomena. Moskow, Mir, 1973 (in Russian).

5. Soldatova E.D., Cond. Matter Physics, 1999, 2, No. 4, 603-616.

6. Soldatova E.D., Snegiryov M.G., Ukraine Journal of Physics, 2001, 46, No. 10, 1111-1115.

7. Soldatova E.D., Snegiryov M.G., Journal of Physical Studies, 2001, 5, No. 1, 24-30 (in Ukrainian).

8. Kurtsar A.R., Physics of Metals and The Physical Metallurgy, 1972, 33, No. 5, 1104-1108 (in Russian).

9. Aptekar I.A., Ponyatovsky E.G., Physics of Metals and The Physical Metallurgy, 1968, 25, No. 5,6, 777-1049 (in Russian).

10. Rheinford B.D., Edwards D.M., Journal of Magnetism and Magnetic Matirials, 1987, No. 63-64, 557559.

11. Johansson B. et al., Phys. Rev. Lett., 1995, 74, 2335-2338.

\section{Деякі особливості асимптотичної поведінки металевого церію}

\author{
Є.Д.Солдатова, Т.Є.Галаченко \\ Дніпропетровський національний університет \\ Отримано 18 липня 2005 р., в остаточному вигляді - \\ 19 грудня 2005 р.
}

На основі вимог термодинамічної стійкості для узагальненої однокомпонентної системи, без за-
лучення будь-яких гіпотез отримані співвідношення між критичними показниками основних хара-
ктеристик стійкості. Знайдені умови, коли ці співвідношення виконуються як рівності. Результати є
застосовані для розгляду критичних властивостей ізоморфного $\gamma \leftrightarrow \alpha$ переходу в металевому церії.
Показано, що для церію відповідні співвідношення виконуються як рівності. Показано також, що лінія
рівноваги на фазовій $P-T$ діаграмі церія безперервно переходить в лінію зниженої стійкості в за-
критичній області, що зумовлює симетрію між його критичними показниками. Отримані результати
відповідають висновкам статичної гіпотези подібності без залучення припущення про однорідність
функції для потенціалів.

Ключові слова: коефіціенти стабільності, критичні показники, гіпотеза подібності, крива фазової рівноваги, квазіспінодаль

PACS: $64.60 . \mathrm{Fr}$ 